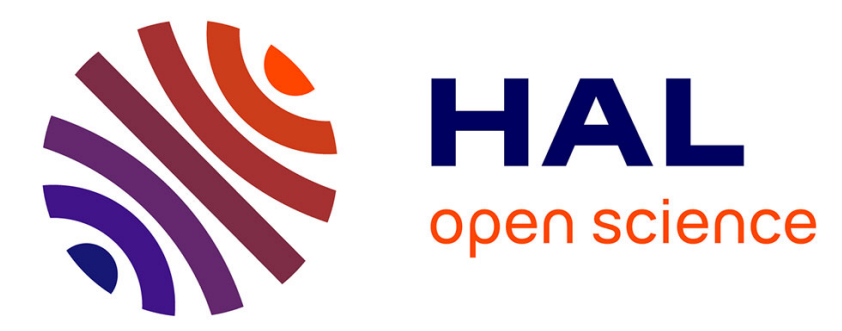

\title{
The ISO 13381-1 Standard's failure prognostics process through an example.
}

Diego Tobon-Meja, Kamal Medjaher, Noureddine Zerhouni

\section{To cite this version:}

Diego Tobon-Meja, Kamal Medjaher, Noureddine Zerhouni. The ISO 13381-1 Standard's failure prognostics process through an example.. IEEE Prognostics \& System Health Management Conference, PHM'2010., Jan 2010, Macau, China. 11 p. hal-00459269

\section{HAL Id: hal-00459269 \\ https://hal.science/hal-00459269}

Submitted on 23 Feb 2010

HAL is a multi-disciplinary open access archive for the deposit and dissemination of scientific research documents, whether they are published or not. The documents may come from teaching and research institutions in France or abroad, or from public or private research centers.
L'archive ouverte pluridisciplinaire HAL, est destinée au dépôt et à la diffusion de documents scientifiques de niveau recherche, publiés ou non, émanant des établissements d'enseignement et de recherche français ou étrangers, des laboratoires publics ou privés. 


\title{
The ISO 13381-1 Standard's Failure Prognostics Process Through an Example
}

\author{
Diego A. Tobon-Mejia, Kamal Medjaher and Noureddine Zerhouni \\ FEMTO-ST Institute, UMR CNRS 6174 - UFC / ENSMM / UTBM \\ Automatic Control and Micro-Mechatronic Systems Department \\ 24, rue Alain Savary, 25000 Besançon, France \\ diego.tobon@femto-st.fr
}

\begin{abstract}
Industrial failure prognostics can be considered as the key process of any condition-based maintenance solution. However, contrary to fault diagnostics which is a mature research and industrial work, failure prognostics is a new field for which few applications exist. In the last decade, the interest for this activity has led to some open and industrial standards where the main objective is to provide users with a guidelines allowing them to perform failure prognostics for a large class of industrial systems. However, these standards, rightly, do not emphasize on any particular example to illustrate their content. The present paper aims at explaining the process of failure prognostics, presented in the standard ISO 13381-1, through an electromechanical example. The purpose is to help beginner researchers in the field of industrial failure prognostics to assimilate the main tasks of the process proposed by the standard. The prognostics process is chosen because it represents the key task among the rest of topics proposed and published by the standard. Thus, the comprehension of this part is important to develop prognostics methods and algorithms based on the solid recommendations given by the international organization for standardization.
\end{abstract}

\section{INTRODUCTION}

The need of reducing maintenance costs, improving the availability and reliability, and reducing or eliminating human and environmental accidents is nowadays a deep wish of any industrial company. To reach these objectives, it is necessary to adopt an appropriate maintenance strategy among the existing ones. Indeed, maintenance can be curative or preventive. For the last case, maintenance tasks are either planned in advance for the systematic one or achieved depending on the real state of the system, which is the case for the Condition-Based Maintenance (CBM). In this maintenance policy $[1,2]$ the system is monitored by a set of sensors which allow to track its state and decide whether a maintenance action is needed or not. To help performing this, failure prognostics seems to be the convenient process that allows to estimate the Remaining Useful Life (RUL) of the system and thus taking appropriate decisions. Contrary to fault detection and isolation (FDI) or fault diagnostics, for which many research and application works are done, failure prognostics research activities are at their infancy stage $[1,3]$. However, this field is gaining more and more interest from both industrial and research communities. During the last decade, many definitions, methods and tools are proposed in the field of failure prognostics, but this is still an open research area [4]. The recent abundance of literature regarding failure prognostics may be a positive point, but it can also be a source of confusion, especially for those who are beginning to work in this domain. The role of any standard, open or not, is then to provide users with some guidelines to help them to accomplish their expected missions. Thus, in the open standard [5] one can find some of the basic elements that should be fulfilled in order to perform failure prognostics. In the same way, the standard ISO 13381-1 [6] defines failure prognostics, details the steps of the prognostics process, gives indications on the monitoring system and on how to estimate the confidence interval associated with the calculated RUL and proposes some mathematical tools which can be used to model the degradation. However, as for any standard, it does not focus on a particular application, neither gives any illustrative example.

The present paper aims at explaining a part of what is published in the standard ISO 13381-1, namely the failure prognostics process. Each step of the process will be progressively explained on an electromechanical example. Section 2 of the paper briefly summarizes the main points addressed in the standard. In section 3 the electromechanical example is presented, followed by the definition of the failure modes given in section 4 . Section 5 is dedicated to the explanation of the prognostics process by using the example presented in the previous section. Finally, a conclusion is given at the end of the paper.

\section{STANDARD ISO 13381-1}

There exists in the literature some discussions about prognostics in the framework of CBM open standards [5, 7-9] proposed by the OpenO\&M initiative [10] but in this paper only the one of the ISO is addressed. The standard published by the ISO deals with the main guidelines and aspects one has to take into account in order to perform failure prognostics on engineering systems. The standard gives the definition of prognostics, specifies the required types of data to consider, presents the concept and the process of prognostics along with all necessary parameters, descriptors and influence factors to consider in order to well estimate the remaining useful life and the associated confidence value of a given system. Note that the standard uses the terminology estimated time to failure (ETTF) instead to RUL. The document also indicates a set of mathematical models which can be used to model the degradation phenomena.

Among the topics presented in the standard, three main aspects can be pinpointed and deserve more attention, namely the required data, the general monitoring process and the prognostics process. In the following, only the failure 
prognostics process is discussed and illustrated on a simple academic example.

\section{SYSTEM DESCRIPTION}

For the explanation of the generic prognostics process proposed by the standard, a degradation phenomenon in an oil pump is considered. Pumping systems were chosen because, in one hand, they are one of the most critical mechanical systems in the industry and, in the other hand they are frequently used in the validation of prognostics models. For example, in [11] oil pumps were used with different contamination levels to test and to validate the data-driven approach the authors have developed and which is based on Hidden Semi-Markov Models (HSMM). In [12] the authors have used the condition and the event data of centrifugal pumps for validation too. Their model was based on a feed-forward neuronal network where training targets were the asset survival probabilities estimated using a variation of the Kaplan-Meier estimator and a degradationbased failure probability density function (pdf) estimator.

For the present paper, the considered example is shown in figure 1. The system is composed of three main components: one induction motor, one hydrostatic bearing and one centrifugal pump (the function of each component and the energy flow is explained below). The system is monitored by different sensors coupled with an acquisition system used to assess the actual condition of the system and to perform its health prognostics.

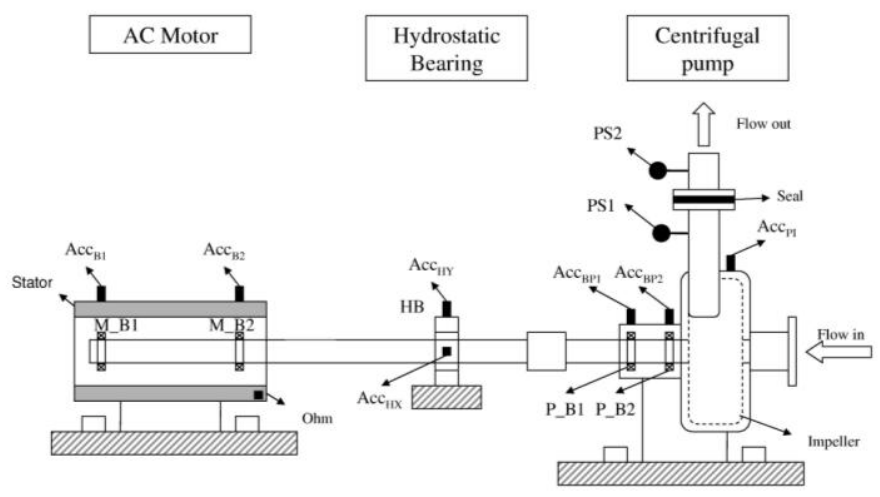

Figure 1: Pumping System.

The main components of the pumping system of figure 1 and their corresponding functions are explained hereafter.

- Induction motor: this system converts the electric energy into a kinetic energy by the interaction of magnetic fields and current-carrying conductors. This device is supplied by an alternating current, and can be separated in two main parts: an outside stationary stator that has coils supplied with alternating current to produce a rotating magnetic field, and an inside rotor guided by bearings linked to the output shaft to generate a mechanical torque by the rotating field.
- Hydrostatic bearing: solely supports the bearing's loads on a thin layer of liquid or gas. Hydrostatic bearings are frequently used in high load, high speed or high precision applications where ordinary ball bearings have short life or high noise and vibration. In our example this bearing is used to guide the shaft toward the pump and to avoid the shaft deflection.

- $\quad$ Centrifugal pump: used to move liquids through a piping system. The fluid enters the pump impeller along or near the rotating axis (which is guided by bearings) and is accelerated by the impeller, radial flowing outward into a diffuser or volute chamber (casing), from where it exits into the downstream piping system. Here, the pump performs the expected or final activity "move a fluid at a constant flow rate and pressure".

- $\quad$ Sensors: used to perform the prognostics and to assess the actual condition of the main components. Three types of sensors are used in the monitoring process: accelerometers which can capture the information contained in the vibration profile, pressure sensors that can monitor with confidence the refueling process in the pump and finally, an ohmmeter to measure the resistance of the stator coils.

Table 1 presents the nomenclature of the used sensors and the monitored components.

The pumping system works under its nominal specifications: the flow rate, the speed and the pressure are assumed to be constant values during the time (it is supposed that the system is well controlled and without any external perturbation). The sensors used in the monitoring process are taken as inputs of suitable acquisition card which allows the data sampling at a constant rate and uses anti-aliasing filters at a correct frequency.

\section{PROGNOSTICS INPUTS}

The first step in building a prognostics system, as published in the ISO standard, is the identification of the set of failure modes (FM), their influence factors on each other and the detection measures (descriptors) that allow to track the evolution of the degradation. The international standard IEC 60812 [13] has presented a procedure named "Procedure for failure mode and effects analysis (FMECA)", which helps the identification of all the failure modes for a specific system, by the analysis of its subsystems and components. Also, the FMECA method classifies the FMs using risk priority numbers (RPN) that are calculated with three failure mode parameters: occurrence (Occ), detection (Det) and severity (Sev). So, the FMECA allows the definition of the appropriate detection method and measures to be used in the diagnostics as well as in the prognostics of the failure modes.

Concerning the pumping system, the results of the corresponding FMECA analysis are shown in tables 2 and 3. For this system, only eight FMs are considered and they are defined as critical. So, the quantification with the RPN was not necessary, because each FM can compromise the ended function. The critical components are: the two bearings and the stator in the electric motor, the oil in the hydrostatic bearing and the two bearings, the impeller and the seal in the 
centrifugal pump. To track the failure mode evolution, different descriptors were defined. Some of these descriptors were tested in the literature and have shown promising results. The failure modes and their corresponding descriptors are defined in the following paragraphs.

- $\quad$ For the bearings failure, the descriptor to be used is the measure of the "health probability". This descriptor is based on wavelet packet decomposition and Hidden Markov Models (HMM) for tracking the severity of bearing faults. In this case, the diagnostics system estimates the probability that the current measures are generated by the normal condition HMM model. So, any decrease of this probability is considered as an indication of an incipient fault (see [14] for more details).

\begin{tabular}{|c|c|c|c|c|}
\hline Abbreviation & Sensor type & Component & Subsystem & System \\
\hline $\mathrm{Acc}_{\mathrm{B} 1}$ & Accelerometer & Bearing 1 & \multirow{3}{*}{ AC Motor } & \multirow{10}{*}{ Pumping System } \\
\hline $\mathrm{Acc}_{\mathrm{B} 2}$ & Accelerometer & Bearing 2 & & \\
\hline Ohm & Ohmeter & Stator & & \\
\hline $\mathrm{Acc}_{\mathrm{HX}}$ & Accelerometer & Shaft & \multirow{2}{*}{ Hydrostatic bearing } & \\
\hline $\mathrm{Acc}_{\mathrm{HY}}$ & Accelerometer & Shaft & & \\
\hline $\mathrm{Acc}_{\mathrm{BP} 1}$ & Accelerometer & Bearing 1 pump & \multirow{5}{*}{ Centrifugal pump } & \\
\hline $\mathrm{Acc}_{\mathrm{BP} 2}$ & Accelerometer & Bearing 1 pump & & \\
\hline $\mathrm{Acc}_{\mathrm{PI}}$ & Accelerometer & Impeller pump & & \\
\hline PS1 & Pressure & \multirow{2}{*}{ Seal } & & \\
\hline PS2 & Pressure & & & \\
\hline
\end{tabular}

Table 1: Sensors, abbreviations and associated monitored componen

- $\quad$ For the stator in the electric motor, the descriptor used is the resistance, it can be measured by a combination of voltage and current measurements or simply by using an ohmmeter (which is the case in this paper) [15].

- The oil degradation in the hydrostatic bearing can be tracked as suggested in [16] by monitoring the center alignment of the shaft. This measure represents the thickness of the oil film.

- The cavitation in the pump which deteriorates the impeller by the implosion of the bubbles can be tracked by the acoustic emissions in the same way as proposed by Escaler [17].

- $\quad$ The leakage is monitored by measuring the differential pressure between the flow before and after the seal and assuming that the only component which can cause a leakage is the seal.

As this is presented in the standard, the initiation criteria of a failure mode must be defined by set of values which alerts the user about a failure mode initiation. For the pumping system, no numerical values were defined as this is considered as an hypothetical example. Moreover, the FMECA analysis sheet proposed for this example allows to record these values. The alarm value is highlighted in yellow in tables 2 and 3 .

In the same way, the standard specifies the definition of other detection values for a failure mode, namely the alert and the trip (shut-down) values. The alert value is higher than the alarm value, but lower than trip value (see Eq. 1). The alert value represents an advanced state of degradation and informs the maintainer that a maintenance task should be defined to assess the real condition and to avoid the break down of the system. In the corresponding FMECA sheet, the space to register this value is also considered and must be defined if this example wants to be replaced by a practical one. The blue column in the FMECA sheet is there to indicate this value. For the alert value definition, several phenomena must be considered as proposed in the standard:

- the confidence level of the prognostics,

- the future production requirements,

- the lead times of spare parts,

- the required maintenance planning,

- the work required to rectify the faults,

- $\quad$ and he trend extrapolation and projection.

$$
\text { alarm }<\text { alert }<\text { trip }
$$

The trip, or the shut-down value, specifies the value that the parameter/descriptor reaches when the component must be fixed without any exception. Normally, this value is lower than the real break value and can be understood as the security factor in strength of materials. This value can be defined from standards, manufacturer's guidelines or by experience. In the pumping system, the FMECA sheet contains also a column to take into account these values, (see the red colored column in tables 2 and 3 ).

\section{PROGNOSTICS PROCESS}

In the same as the ISO, some researchers and industrials are aware of the importance of prognostics in the CBM policy and this pushed them to make some efforts in their standardization. For example, the cooperation between scientists and industrials has enabled the development of a standard platform to build a CBM software [9], called "Open Systems Architecture for Condition-based Maintenance". In this platform the 
standardization of the prognostics process is discussed [7]. The previous standards and the ISO standard, are consistent in different points, especially in the fact that the prognostics is a process which comes after the monitoring process. This is because the monitoring process is used to estimate the actual health state of the system and also to generate data which are useful for the prognostics process.

The ISO standard defines the prognostics as a sequential process (see figure 2) with four main steps:

1. Pre-processing: at this step the system identifies all the existing failure modes, their relations, symptoms parameters - descriptors and determines the potential future failure modes.

2. Existing failure mode prognostics process: a study of all existing failure modes is then performed, the severity and the Estimated Time To Failure (ETTF) are calculated.

3. Future failure mode prognostics process: the most probable future modes, the influence factors between them and the existing modes are estimated. Also, the ETTF for the future failure modes is calculated.

4. Post-action prognostics: in this step the prognostics system proposes the maintenance actions to be done in order to avoid, reduce or delay the failure mode effects. After this, a new prognostics is made by taking into account the suggested actions, and the confidence about the estimated time to failure is calculated.

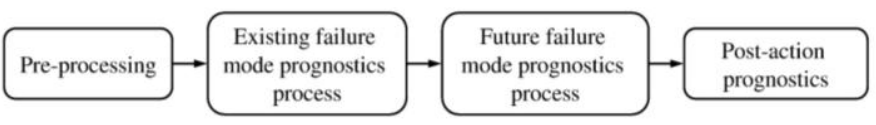

Figure 2: Pumping System.

In the following, the pumping system presented previously is used to explain the main steps of the prognostics process.

\section{Pre-processing}

During this step the following operations are made:

1. The first step deals with diagnostics to identify all the failure modes which exist at a present time in the pumping system. In our case a Hidden Markov Model can be made for each failure mode listed in the FMECA sheet as proposed in [18] to detect the existing failure modes. For the studied pumping system the result shown in figure 3 can be obtained. In this application example, the existing failures are:

- Two growing outer race defects in the bearing 1 of the motor and in the bearing 2 of the pump. Both failure modes come from the past. They are in fact an evolved version of past failure modes. For this reason the diagnostics system places these failure modes in the past line.

- Two new failure modes that the diagnostics system has identified at the present iteration time, by using the last monitored data of the descriptors D4 and D8. These failure modes are an oil degradation in the hydrostatic bearing and a leakage in the seal of the pumping system.
2. The second step consists in the identification of the influence factors between the existing failure modes. To perform this operation the prognostics system can use the "effects on" column in the FMECA analysis (see tables 2 and 3 ) to find the relations between the FMs. Once this operation is performed for the pumping system, by using the diagnostic

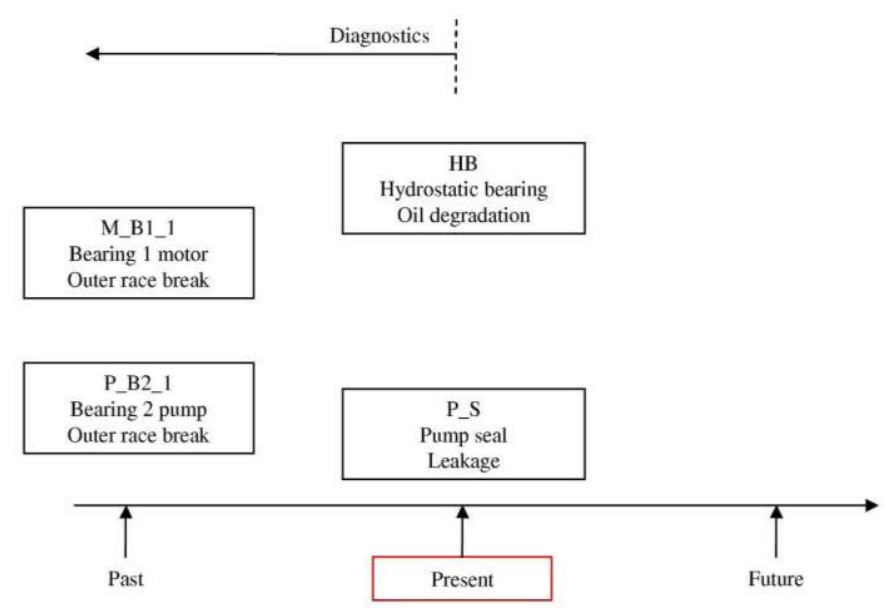

Figure 3: Pumping System.

results obtained in the previous step and the FMECA analysis, the prognostics system is able to define the relations displayed with orange color in figure 4 .
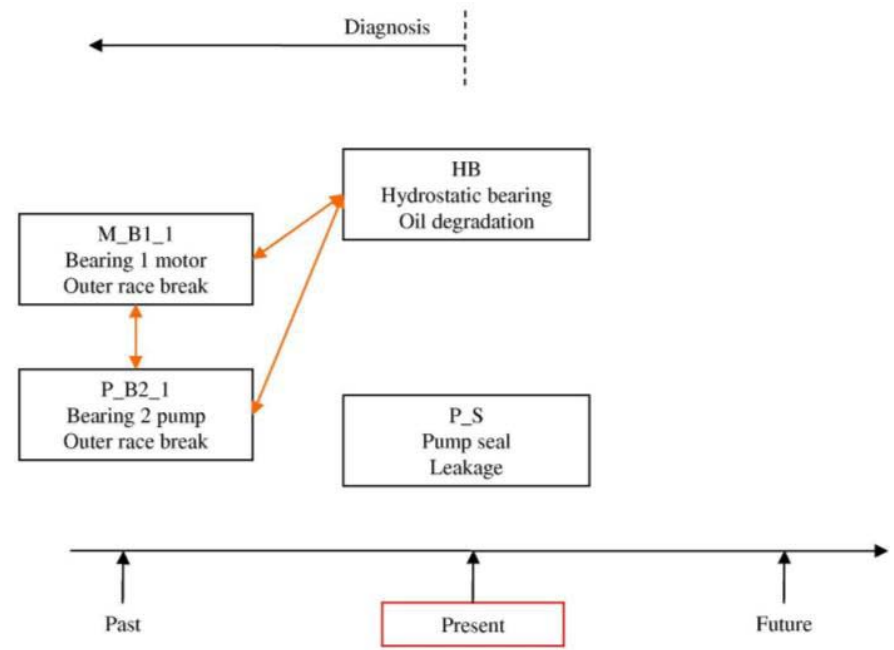

Figure 4: Existing relations between the failure modes

3. Once the existing failure modes and their relations are identified, the system has to retrieve all the information available about these failure modes. In the pumping system, the FMECA analysis (table 2 and table 3 ) has different detection levels (alarm, alert, trip). The prognostics system must then retrieve this information to track the degradation. For example, for the descriptor "D1" which is used 


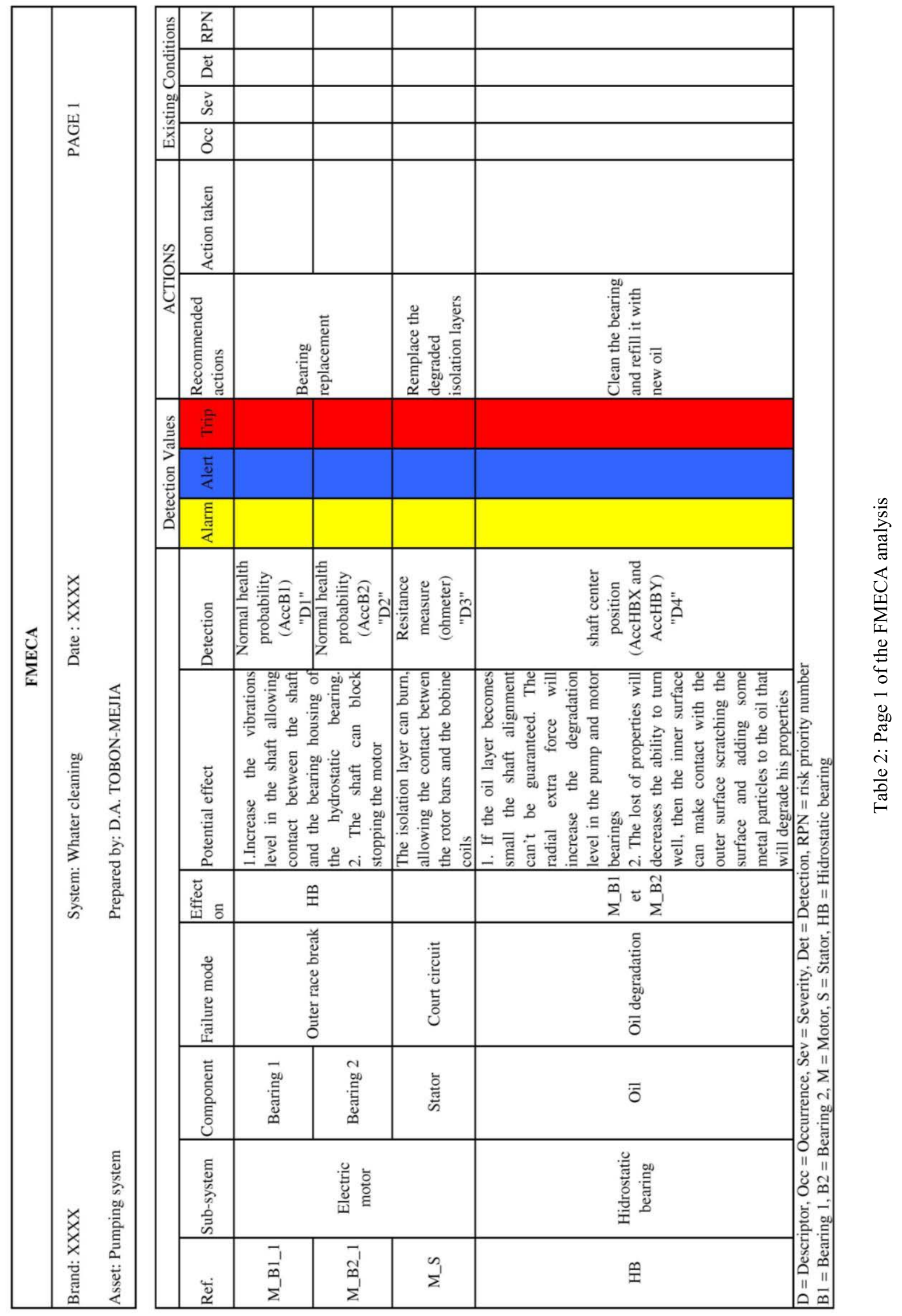




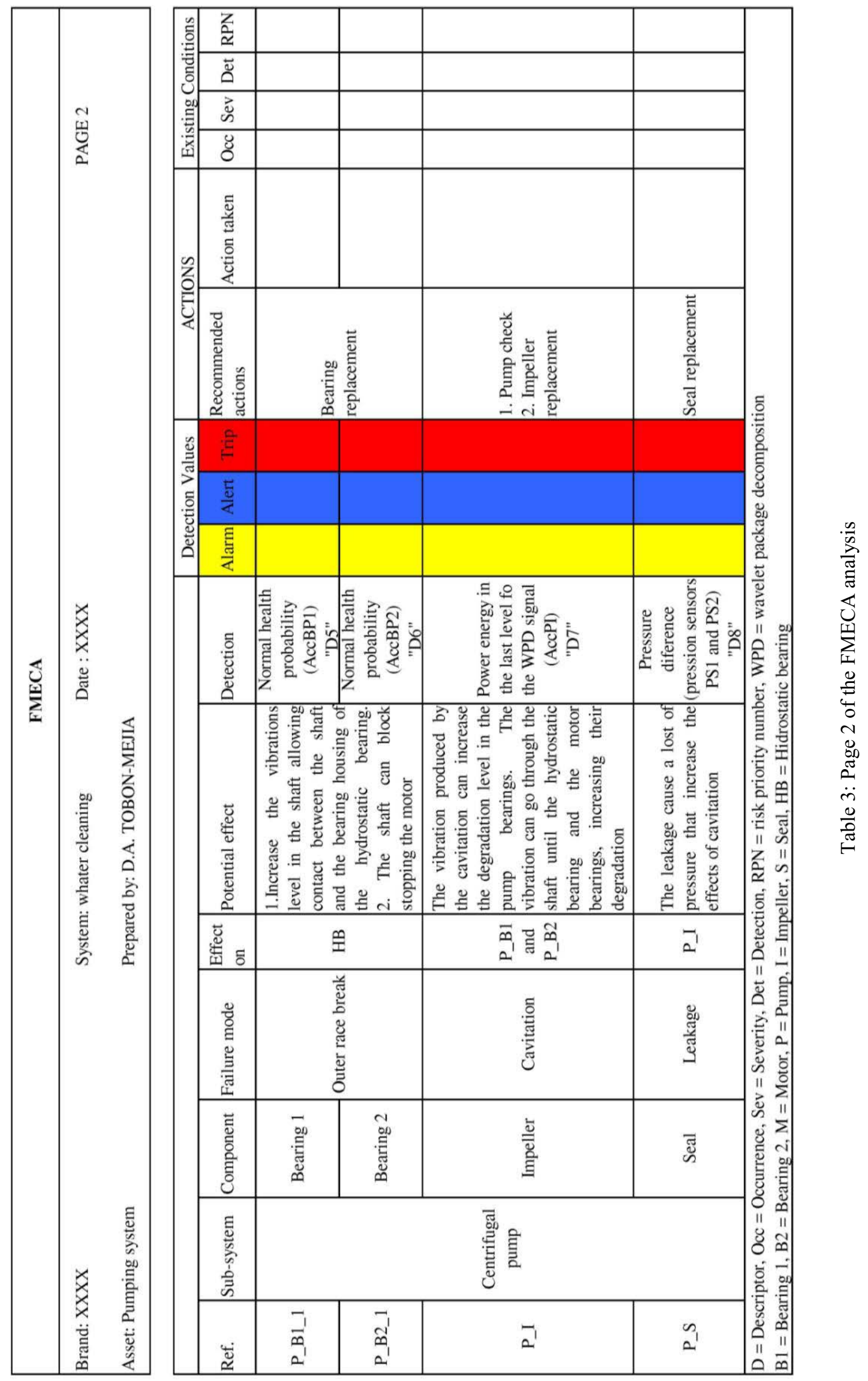


to track the outer race break, the system registers the initiation criteria value (alarm), the alert value, the trip value and the actual value as shown in figure 5. This operation must be made for all the descriptors of all the existing failure modes.

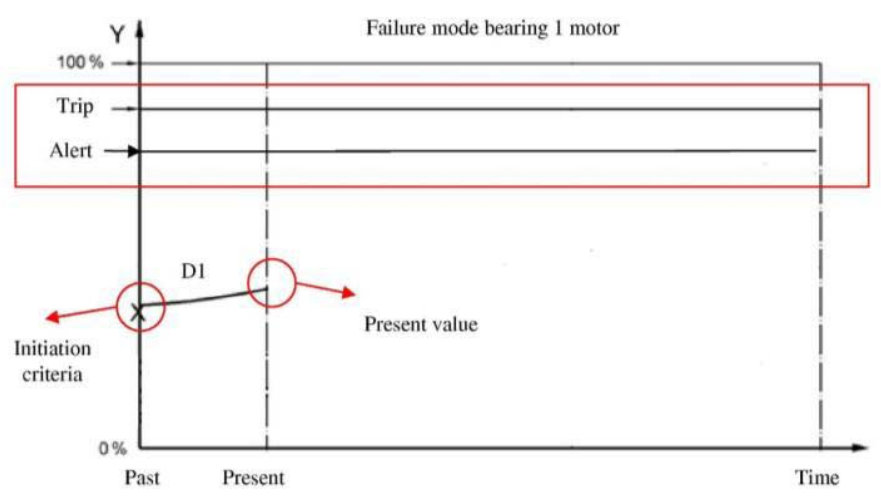

Figure 5: Existing failure mode values.

4. The last step in the pre-processing process consists in determining the potential future failure modes, their initiation criteria and the failure definition set points. For example, in the pumping system the old maintenance histories data can be used to find the most probable future failure modes. For this, the prognostics system can proceed by similarity study and analysis (similar diagnosed failure modes with their associated monitoring values) on the previous recorded situations to find the potential future failure modes. The result of this step is shown in the green box of figure 6 .

\section{Existing failure modes prognostics process}

During this step the following operations are made:

1. The first step of this process aims at identifying the actual condition of the overall system, and the severity of the existing failure modes. For this, the prognostics system will classify the failure modes from the most severe to less severe. To perform this quantification, the system measures the distance between the actual descriptor value with its respective trip value, and the smallest distance defines the most sever failure mode. For example, in the pumping system if the prognostics system displays in the same window (using scales factors) the descriptors "D1" and "D4" which have the trends showed in the figure 7 , the system concludes that the outer race break FM in the bearing 1 of the AC motor (M_B1_1) is more severe at the present time than the oil degradation FM in the hydrostatic bearing (HB). This is because the distance between its current value and its trip value is shorter than the descriptor "D4".

2. The next step is the time projection of the parameters/descriptors into the future. For this purpose, the standard presents projection and extrapolation as trending tools. The basic difference between trend extrapolation and trend projection is that projection requires the estimation of

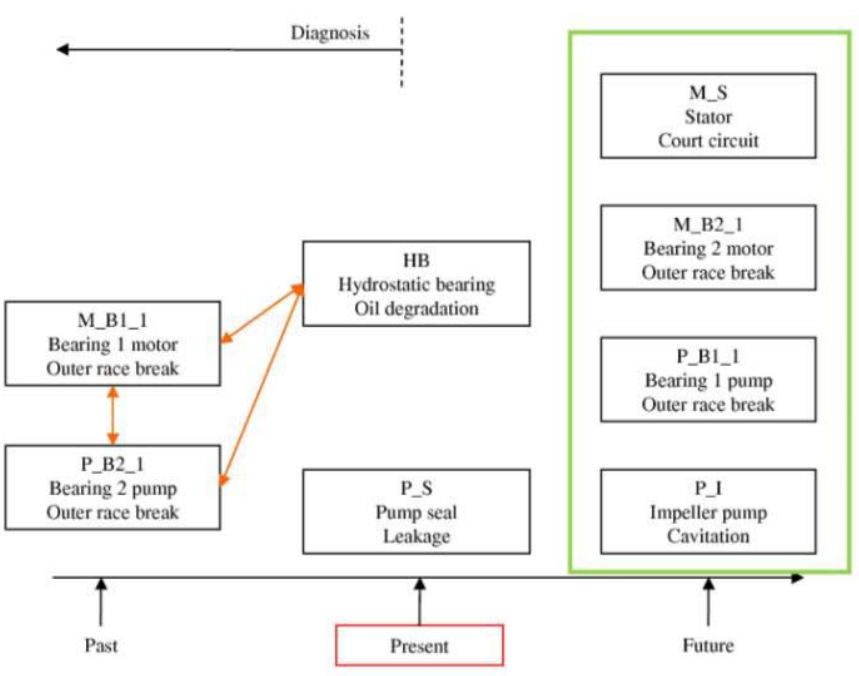

Figure 6: Probable failure mode values.

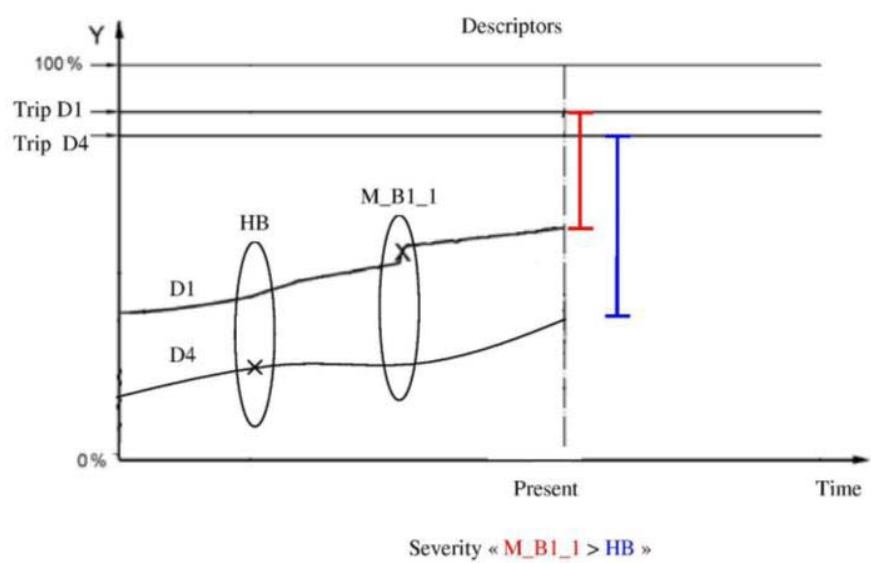

Figure 7: Severity of existing failure modes.

extrapolation curve is fitted only to the existing data. The projection is then more accurate than the extrapolation, if the system disposes enough information or is accurately modeled and captures all the failure modes behavior. Interested readers can refer to the reviews about several prognostics approaches and methods published in [1] and in [19]. In the example of the pumping system, linear regression models coupled with past information can be used to project the descriptors as proposed in [20] or in [21]. For example for the descriptors "D1" and "D4" the system will trend a curve like the dotted lines shown in figure 8 .

3. The subsequent step consists in a simple analysis of the projection curves. The prognostics system estimates when the projected parameter/descriptor reaches its respective trip value (failure), and estimates the difference between the actual time and the future failure time. This time difference is the estimated time to failure "ETTF". In the case of the pumping system, the prognostics model makes the 
intersection between the projected values and the trip value defined for all failure modes in the FMECA sheet and finds the shortest "ETTF". For example, in figure 8 the prognostics system projected the descriptors D1 and D4, and it is possible to see that the descriptor "D4" reaches first its trip value, whereas the descriptor "D1" does not reach its corresponding trip value. Then, the system will keep in memory the "ETTF HB" (green circle in figure 8) which is associated with the failure mode "HB" (oil degradation).

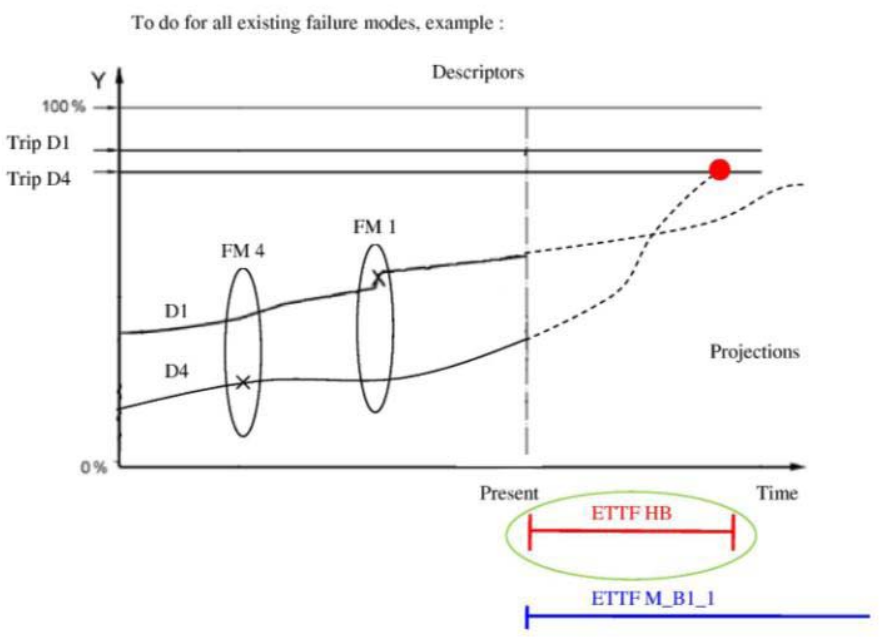

Figure 8: Parameters/descriptors projection and ETTF.

4. Finally, the prognostics process must assure that the projection is under the specified confidence level. In the pumping system, to calculate the confidence interval, it is possible to use for example the Monte Carlo method to take into account the variation of the parameters' values as in [22] to estimate the ETTF of a suspension system.

\section{Future failure modes prognostics process}

1. The first operation in this step is the determination of the most probable future failure modes. To perform this operation the prognostics system can use the initiation criteria values and the projections presented in the previous subsection. The system will make the intersection between the "alarm" values of the potential failure modes obtained in the step 4 of subsection "Pre-processing". Then the failure modes which drop in this class will be retained as the most probable future failure modes, whereas the others will be removed for the current iteration. In the pumping system it is possible to find a situation as presented in figure 9 , where the descriptor projection does not reach the initiation criteria of the short-circuit in the stator (M_S). So, this failure mode is then removed and only three future failure modes are retained and handled, as shown in the red box of figure 9 .

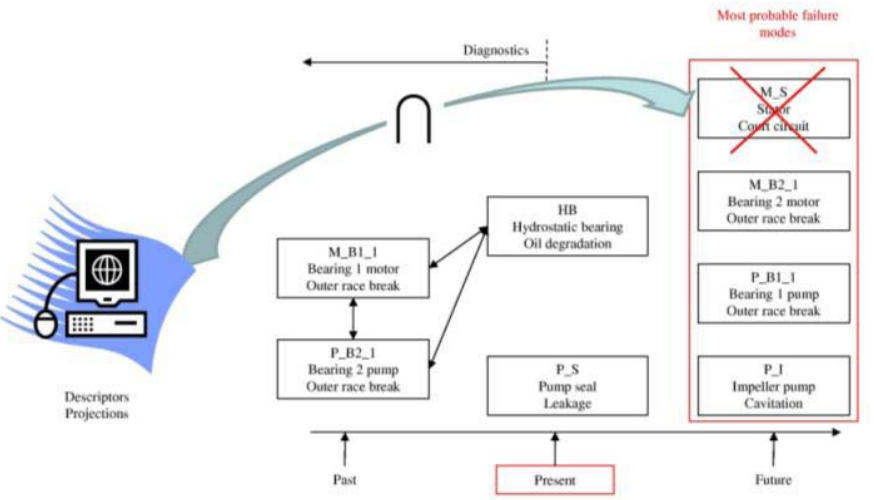

Figure 9: Most probable failure mode estimation.

2. The next step is similar to the step 2 of subsection "Pre-processing" where the influence factors were estimated. Here the system estimates the influence factor between all the failure modes (existing and future ones). For the pumping system, knowing the existing and the future failure modes calculated in the previous step, the system can use the information indicated in the FMECA sheet ("effect on" column) to determine the influence between all the failure modes. Figure 10 shows the influence factors in the studied pumping system, the orange arrows represent the influence between the existing failure modes, the red arrows represent the influence between future failure modes and the green arrows identify the relations between existing and future failure modes. Some arrows are bidirectional meaning that degradation in one component may affect directly the other and vice versa. This phenomenon is important and has to be taken into account because a failure mode which evolves faster and which is near the trip value will degrade the related components too.

3. This step is similar to steps 2 to 4 presented in subsection "Existing failure modes prognostics process", in where the system estimates the ETTF for the future failure modes. To do this, the prognostics system will verify the projections by considering the influence factors, the trip values and the actual descriptor value.After this, the system will estimate and retain the shortest future ETTF. In the pumping system some descriptors of the probable future failure mode obtained at step 1 of subsection "Future failure modes prognosis process" may reach their trip values. For example the descriptor "D7" shown in figure 11 and which tracks the degradation in the impeller of the pump will reach the failure state. So, an ETTF can be estimated and corresponds to the shortest future ETTF which must be stored in memory. For the case presented in figure 11 the "ETTF P_I" will be retained (green circle in figure 11). 


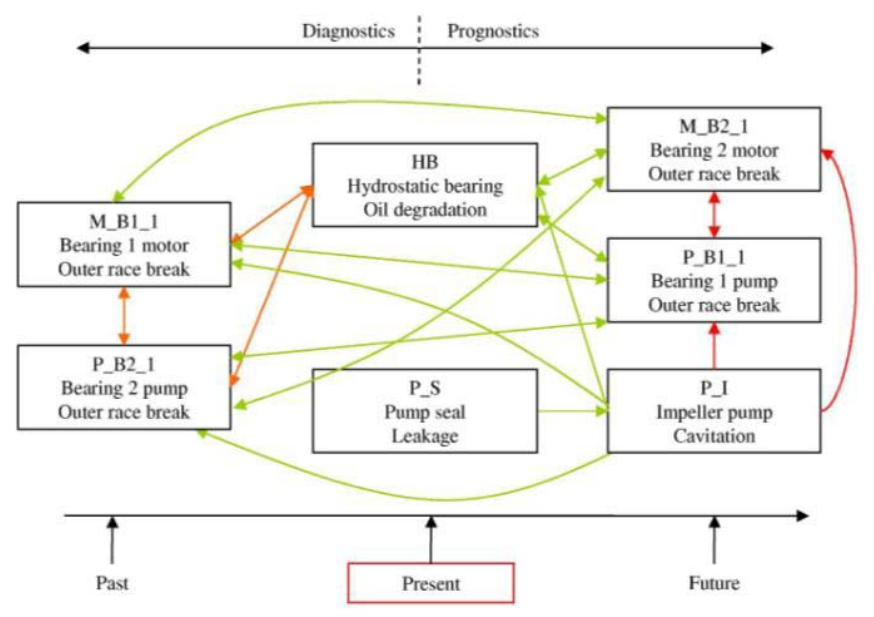

Figure 10: Influence factors between all the FMs.

\section{Post-action prognostics}

1. Here, the system will identify one or more maintenance actions which may delay, stop or eliminate the progression of the critical existing failure mode and prevent the initiation of future failure modes. For the pumping system, these actions are recommended in the FMECA sheet. From the previous steps two critical failure modes with short ETTF were identified, they are the "ETTF_HB" and the "ETTF P_I". The prognostics system will then suggest to perform an oil renewal and a pump inspection or an impeller replacement. These actions are aimed at avoiding the failure modes and eliminating their influence on the other failure modes as shown in figure 12.

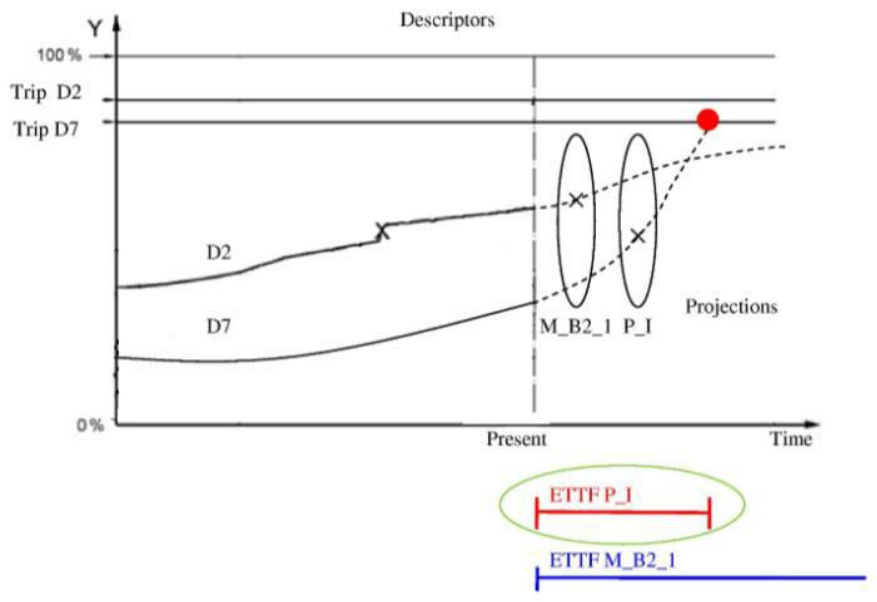

Figure 11: ETTF fo the future FMs.

2. At this step the system will re-estimate the existing and the future failure modes by taking into account the proposed maintenance actions. For the pumping system example, after having done the proposed actions the prognostics system will store all the failure modes (future and existing) in memory. They will then be used in the next iteration as existing failure modes and their presence will be validated or invalidated by the diagnostics system.
3. Finally, the prognostics system will present to the maintainer the results, which are: the critical FMs and their corresponding ETTFs, the maintenance actions, the confidence values and the validity conditions. The standard suggests many error sources which can affect the accuracy of the calculated ETTFs and confidence values. For example, confidence values can be completely different for each descriptor because they depend on many parameters, like the quality and the quantity of available information, the nature of the database, the data acquisition and processing methods, etc. For the pumping system example, the prognostics system will present to the maintainer two messages, namely:

a) An oil degradation is present in the hydrostatic bearing, the ETTF is "ETTF HB" with a confidence value of "Y" $\%$, an oil replacement is recommended. This prognosis is valid under the assumption that the production plan is the "Production" plan and the weather conditions will stay as specified in the "Weather" file.

b) The actual conditions and the monitoring history advise the possibility of cavitation which will degrade the impeller; a pump inspection or an impeller replacement is then recommended with an associated confidence value of "R" \%. This prognosis result is valid under the assumption that the production plan is the "Production" plan and the weather conditions will stay as specified in the "Weather" file.

\section{Conclusions}

Many interesting topics are presented in the ISO 13381-1, such as the condition monitoring flow chart detailing the prognostics process, the prognostics basic concepts and the influence factors. The prognostics process presented in the standard has retained the attention because it can be considered as the key process of any CBM solution. The generic process presented by the standard may allow the users to understand, develop and make failure prognostics if the appropiated means are used.

The example presented in this paper helps the new users interested in industrial prognostics to understand in an easy way the process suggested by the standard. Indeed, the prognostics process as presented by the ISO is ambiguous in many topics like for example the concept of existing failure modes and the evolution of past failure modes, which are not explained in detail. Thus, new users can have problems in understanding the prognostics process.

The work presented in this paper aimed at explaining as well as possible the prognostics process of the ISO 13381-1 standard through an example. However, the paper did not give any numerical quantification of this ETTF or about the corresponding confidence interval. This paper did not also use any specific modeling or projection mathematical tool, because an hypothetical example was used, but some references about appropriated projections methods are given. The choice of appropriate modeling and projection tools for RUL (or ETTF) and confidence interval estimation depends in fact on the type 

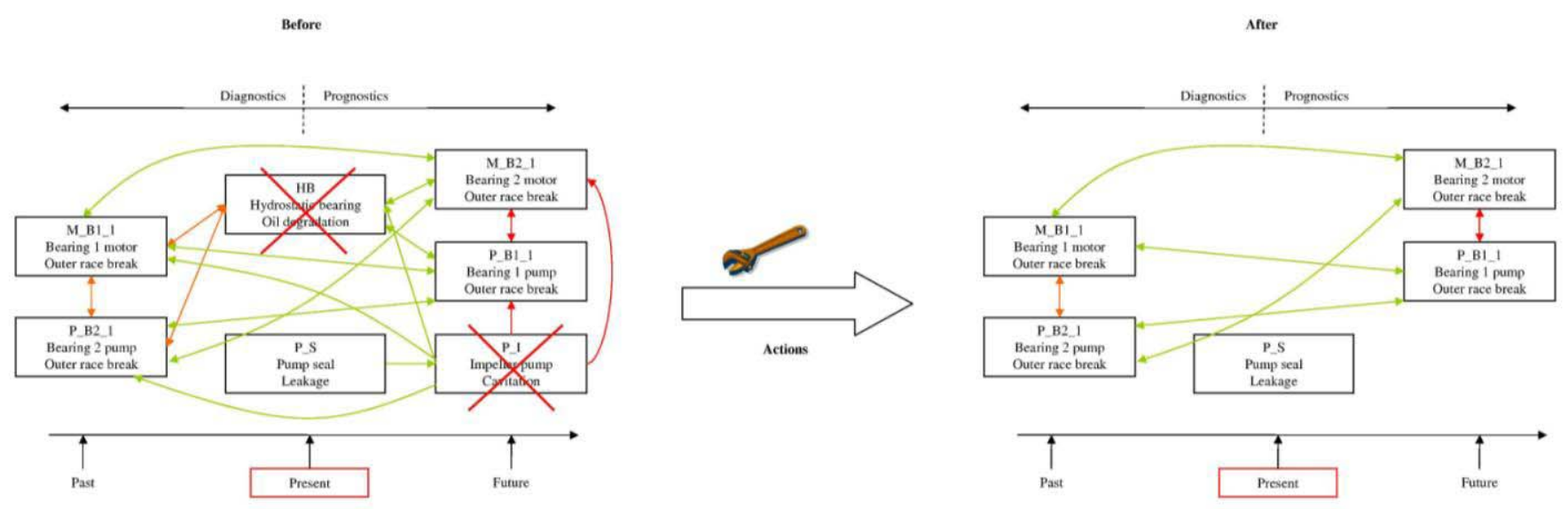

Figure 12: Maintenance actions and their effects on the FMs.

of the available data, the degradation mechanisms, the the complexity of the system, the prognostics precision, and on many other environmental factors. All these points are some of challenges that deserve to be developed in the future works.

\section{References}

1. Andrew K.S. Jardine, Daming Lin, and Dragan Banjevic. A review on machinery diagnostics and prognostics implementing condition-based maintenance. Mechanical Systems and Signal Processing, 20(7):1483 - 1510, 2006.

2. G. Vachtsevanos, F.L. Lewis, M. Roemer, A. Hess, and B. $\mathrm{Wu}$. Intelligent Fault Diagnosis and Prognosis for Engineering Systems. Systems. New Jersey, Hoboken: Wiley \& Sons, 2006.

3. Venkat Venkatasubramanian. Prognostic and diagnostic monitoring of complex systems for product lifecycle management: Challenges and opportunities. Computers \& Chemical Engineering, 29(6):1253 - 1263, 2005.

4. Aiwina Heng, Sheng Zhang, Andy C.C. Tan, and Joseph Mathew. Rotating machinery prognostics: State of the art, challenges and opportunities. Mechanical Systems and Signal Processing, 23(3):724 - 739, 2009.

5. M.G. Thurston. An open standard for web-based condition-based maintenance systems. In AUTOTESTCON Proceedings, 2001. IEEE Systems Readiness Technology Conference, pages 401 - 415, 2001.

6. ISO. Condition monitoring and diagnostics of machines prognostics - Part 1: General guidelines. Int. Standard ISO13381-1, 2004.

7. M. Lebold and M. Thurston. Open standards for conditionbased maintenance and prognostic systems. $5^{\text {th }}$ annual maintenance and reliability conference (marcon 2001). Gatlinburg, USA, 2001.

8. J.W. Sheppard, M.A. Kaufman, and T.J. Wilmering. IEEE standards for prognostics and health management. In AUTOTESTCON, 2008 IEEE, pages 97 - 103, 2008.

9. Applied Research Laboratory Penn State University, The Boeing Company, and Machinery Information
Management Open Standards Alliance MIMOSA. Open systems architecture for condition-based maintenance OSA - CBM, 2006.

10. OpenO\&M. http://www.openoandm.org/, 2009.

11. Ming Dong and David He. A segmental hidden semimarkov model (hsmm)-based diagnostics and prognostics framework and methodology. Mechanical Systems and Signal Processing, 21:2248 - 2266, 2006.

12. Aiwina Heng, Andy C.C. Tan, Joseph Mathew, Neil Montgomery, Dragan Banjevic, and Andrew K.S. Jardine. Intelligent conditionbased prediction of machinery reliability. Mechanical Systems and Signal Processing, 23(5):1600 - 1614, 2009.

13. Analysis techniques for system reliability - procedure for failure mode and effects analysis (FMEA), 2006.

14. Hasan Ocak, Kenneth A. Loparo, and Fred M. Discenzo. Online tracking of bearing wear using wavelet packet decomposition and probabilistic modeling: A method for bearing prognostics. Journal of sound and vibration, 302:951 - 961, 2007.

15. Peter Vas. Parameter Estimation, Condition Monitoring, and Diagnosis of Electrical Machines. Clarendon Press, 1993.

16. Agnieszka Muszynska. Rotordynamics. Taylor \& Francis Group, 2005.

17. Xavier Escaler, Mohamed Farhat, Philippe Ausoni, Eduard Egusniza, and Francois Avellan. Cavitation monitoring of hydroturbines: Test in a francis turbine model. In Sixth international symposium on cavitation, 2006.

18. C. Kwan, X. Zhang, R. Xu, andL. Haynes. A novel approach to fault diagnostics and prognostics. In International Conference on Robotics \& Automation, pages $604-609,2003$.

19. H.C. Pusey and M.J. Roemer. An assessment of turbomachinery condition monitoring and failure prognosis technology. The Shock and Vibration Digest, 31:365 - 371, 1999. 
20. Y.G. Li and P. Nilkitsaranont. Gas turbine performance prognostic for condition-based maintenance. Applied Energy, 86(10):2152 - 2161, 2009.

21. Tianyi Wang, Jianbo Yu, David Siegel, and Jay Lee. A similarity-based prognostics approach for remaining useful life estimation of engineered systems. In International conference on prognostics and health management, 2008.

22. Jianhui Luo, Krishna R. Pattipati, Liu Qiao, and Shunsuke Chigusa. Model-based prognostic techniques applied to a suspension system. Transactions on Systems, Man, and Cybernetics, 38:1156 - 1168, 2003.

\section{Biography}

Diego A. TOBON-MEJIA was born in Medellin, Colombia, on February 16, 1985. He received the B.Sc. and S.M., both in 2008, from the National Engineering School in Metz (France) and the EAFIT University (Colombia). He made a specialization in his last school year in research and development in "Design, industrialisation and innovation" supported by the Paul Verlaine University, the ENSAM and the ENIM, all in Metz (France).

In his last year of Master he developed a decision support application based in fuzzy logic and the PERT method to assess the failure mode hazard in a Francis turbine that works in a hydroelectric power station in Colombia, to ensure the systems availability and security. Actually he performs his doctoral studies at Franche-Comté University, in Besançon (France) sponsored by Alstom Transport where he his a research engineer. $\mathrm{He}$ is engaged in research on rotating machinery failure prognostic at the FEMTO-ST institute and Alstom transport.

Diego A. TOBON-MEJIA, was honored in 2002 with the "Excellence scholarship" from the "EEPP de Medellin" (Medelln Public Enterprises) to perform his studies in Colombia. In 2006 he was honored by the The French Ministry of Foreign affairs with the "Eiffel Excellence scholarship" to continue his studies in France.

Dr. Kamal MEDJAHER is an associate Professor at the French high school of mechanics and micro-techniques in Besançon since September 2006, where he teaches control and fault diagnostics and prognostics. After an engineering degree in electronic, he has got his MS in control and industrial computing in 2002 at the "Ecole Centrale de Lille" and his PhD in 2005 in the same field from the University of Lille 1. Before joining his current position, Dr. Medjaher has mainly worked in the field of Fault Detection and Isolation (FDI). He has particularly used the bond graph formalism to derive mathematical models of physical systems and to generate Analytical Redundancy Relations (ARRs) and residuals, which are then used to detect and to isolate possible faults on the system.

Since September 2006, Dr. Medjaher leads research works in the field of failure prognostics and uses artificial intelligent tools, particularly Dynamic Bayesian Networks (DBNs). Dr.
Medjaher co-authored eight published journal papers and more than ten refereed conference papers.

Professor Noureddine ZERHOUNI received his engineer degree from National Engineers and Technicians School of Algiers (ENITA) in 1985. After a short period in industry as engineer, he received his Ph.D. Degree in Automatic Control from the Grenoble National Polytechnic Institute in 1991. In September 1991, he joined the National Engineering School of Belfort (ENIB) as Associate Professor. At this time, his main research activity was concerned with modelling, analysis and control of manufacturing systems.

Since September 1999, Noureddine Zerhouni is Professor at the national high school of mechanics and microtechniques of Besançon. He founded and is responsible of the research group "Design and maintenance of mechatronic systems" (COSMI) of AS2M Department within FEMTO-ST Institute. His main research activities are concerned with intelligent maintenance systems and e-maintenance. Professor Noureddine Zerhouni has been and is involved in various European and National projects on intelligent maintenance systems like FP5 European Integrated Project of ITEA program (Information Technology for European Advancement) PROTEUS, NEMOSYS (Naval EMaintenance Oriented SYStem) with DCNS, and AMIMACFAME (Reliability Improvement of Embedded Machines) with ALSTOM and CEGELEC. 